\title{
LOCAL HOMOLOGY AND GORENSTEIN FLAT MODULES
}

\author{
FATEMEH MOHAMMADI AGHJEH MASHHAD AND KAMRAN DIVAANI-AAZAR
}

\begin{abstract}
Let $R$ be a commutative Noetherian ring, $\mathfrak{a}$ an ideal of $R$ and $\mathcal{D}(R)$ denote the derived category of $R$-modules. We investigate the theory of local homology in conjunction with Gorenstein flat modules. Let $X$ be a homologically bounded to the right complex and $Q$ a bounded to the right complex of Gorenstein flat $R$ modules such that $Q$ and $X$ are isomorphic in $\mathcal{D}(R)$. We establish a natural isomorphism $\mathbf{L} \Lambda^{\mathfrak{a}}(X) \simeq \Lambda^{\mathfrak{a}}(Q)$ in $\mathcal{D}(R)$ which immediately asserts that $\sup \mathbf{L} \Lambda^{\mathfrak{a}}(X) \leq \operatorname{Gfd}_{R} X$. This isomorphism yields several consequences. For instance, in the case $R$ possesses a dualizing complex, we show that $\operatorname{Gfd}_{R} \mathbf{L} \Lambda^{\mathfrak{a}}(X) \leq \operatorname{Gfd}_{R} X$. Also, we establish a criterion for regularity of Gorenstein local rings.
\end{abstract}

\section{Introduction and Prerequisites}

Throughout this paper $R$ is a commutative Noetherian ring and $\mathcal{D}(R)$ denotes the derived category of $R$-modules. The full subcategory of homologically bounded complexes is denoted by $\mathcal{D}_{\square}(R)$ and that of complexes homologically bounded to the right (resp. left) is denoted by $\mathcal{D}_{\sqsupset}(R)$ (resp. $\mathcal{D}_{\sqsubset}(R)$ ). Also, $\mathcal{D}_{\square}^{f}(R)$ stands for the full subcategory of homologically bounded complexes with finitely generated homology modules. We use the symbol $\simeq$ for denoting isomorphisms in the category $\mathcal{D}(R)$. For any complex $X$ in $\mathcal{D}_{\sqsupset}(R)$ (resp. $\mathcal{D}_{\sqsubset}(R)$ ), there is a bounded to the right (resp. left) complex $U$ of projective (resp. injective) $R$-modules such that $U \simeq X$. A such complex $U$ is called a projective (resp. injective) resolution of $X$. We say that a homologically bounded complex $X$ has finite projective (resp. injective) dimension if in $\mathcal{D}(R)$ it is isomorphic to a bounded complex of projective (resp. injective) $R$-modules. The left derived tensor product functor $-\otimes_{R}^{\mathbf{L}} \sim$ is computed by taking a projective resolution of the first argument or of the second one. Also, the right derived homomorphism functor $\mathbf{R} \operatorname{Hom}_{R}(-, \sim)$ is computed by taking a projective resolution of the first argument or by taking an injective resolution of the second one.

Let $\mathfrak{a}$ be an ideal of $R$ and $\mathcal{C}_{0}(R)$ denote the full subcategory of $R$-modules. It is known that the $\mathfrak{a}$-adic completion functor

$$
\Lambda^{\mathfrak{a}}(-)=\lim _{n}\left(R / \mathfrak{a}^{n} \otimes_{R}-\right): \mathcal{C}_{0}(R) \rightarrow \mathcal{C}_{0}(R)
$$

is not right exact in general. The left derived functor of $\Lambda^{\mathfrak{a}}(-)$ exists in $\mathcal{D}(R)$, and so for any complex $X \in \mathcal{D}_{\sqsupset}(R)$, the complex $\mathbf{L} \Lambda^{\mathfrak{a}}(X) \in \mathcal{D}_{\sqsupset}(R)$ is defined by $\mathbf{L} \Lambda^{\mathfrak{a}}(X):=\Lambda^{\mathfrak{a}}(P)$, where $P$ is a (every) projective resolution of $X$. Let $X \in \mathcal{D}_{\sqsupset}(R)$. For any integer $i$, the $i$-th local homology module of $X$ with respect to $\mathfrak{a}$ is defined by $H_{i}^{\mathfrak{a}}(X):=H_{i}\left(\mathbf{L} \Lambda^{\mathfrak{a}}(X)\right)$. The study of local homology modules was initiated by Matlis [M]. Then it was continued by many authors, see e.g. [Si], GM], [LLT], $[\underline{\mathrm{Sc}}$ and $[\mathrm{Fr}$. Let $\check{C}(\underline{\mathfrak{a}})$ denote the $\check{C}$ ech complex of $R$ on a set $\underline{\mathfrak{a}}$ of generators of $\mathfrak{a}$. By [LLT, (0.3),aff,p.4] (see also [Sc, Section 4] for corrections),

$$
\mathbf{L} \Lambda^{\mathfrak{a}}(X) \simeq \mathbf{R} \operatorname{Hom}_{R}(\check{C}(\underline{\mathfrak{a}}), X) .
$$

2000 Mathematics Subject Classification. 13D05, 13D25.

Key words and phrases. Gorenstein flat dimension; large restricted flat dimension; left derived functors; local homology modules. 
By using this isomorphism Frankild [Fr, Theorem 2.11] proved that $\inf \mathbf{L} \Lambda^{\mathfrak{a}}(X)=\operatorname{width}_{R}(\mathfrak{a}, X)$, where $\operatorname{width}_{R}(\mathfrak{a}, X):=\inf \left(R / \mathfrak{a} \otimes_{R}^{\mathbf{L}} X\right)$. Finding a good upper bound for $\sup \mathbf{L} \Lambda^{\mathfrak{a}}(X)$ was considered in $\underline{\mathrm{Sc}}$. and $[\mathrm{Fr}$. The study of connections between Gorenstein injective modules and local cohomology modules was started by Sazeedeh $[\mathrm{Sa}$. Here, we investigate connections between Gorenstein flat modules and local homology modules. The notion of Gorenstein flat modules was introduced by Enochs, Jenda and Torrecillas in [EJT]. An $R$-module $T$ is said to be Gorenstein flat if there exists an exact complex $F$ of flat $R$-modules such that $T \cong \operatorname{im}\left(F_{0} \longrightarrow F_{-1}\right)$ and $F \otimes_{R} I$ is exact for all injective $R$-modules $I$. The Gorenstein flat dimension of $X$ is defined by

$$
\operatorname{Gfd}_{R} X \quad:=\inf \left\{\sup \left\{l \in \mathbb{Z} \mid Q_{l} \neq 0\right\} \mid Q\right. \text { is a bounded to the right complex of }
$$$$
\text { Gorenstein flat R-modules and } Q \simeq X\} \text {. }
$$

For more details on the theory of Gorenstein homological dimensions for complexes, we refer the reader to [C].

Let $T$ be a Gorenstein flat $R$-module and $X \in \mathcal{D}_{\sqsupset}(R)$. We show that $T$ is $\Lambda^{\mathfrak{a}}$-acyclic and $H_{0}^{\mathfrak{a}}(T) \cong \Lambda^{\mathfrak{a}}(T)$. Using this, we prove that if $Q$ is a bounded to the right complex of Gorenstein flat $R$-modules such that $Q \simeq$ $X$, then $\mathbf{L} \Lambda^{\mathfrak{a}}(X) \simeq \Lambda^{\mathfrak{a}}(Q)$, in particular $\sup \mathbf{L} \Lambda^{\mathfrak{a}}(X) \leq \operatorname{Gfd}_{R} X$. We deduce several applications. We show that the large restricted flat dimension of $\Lambda^{\mathfrak{a}}(T)$ is zero. Now, assume that $R$ possesses a dualizing complex. Then, we prove that $\Lambda^{\mathfrak{a}}(T)$ is Gorenstein flat. Also, we establish the inequality $\operatorname{Gfd}_{R} \mathbf{L} \Lambda^{\mathfrak{a}}(X) \leq \operatorname{Gfd}_{R} X$, which improves $[\mathrm{CFH}$, Theorem $5.10 \mathrm{~b})]$. Suppose that $X$ is homologically bounded and let $Y \in \mathcal{D}_{\square}^{f}(R)$ be a non-exact complex. If either projective or injective dimension of $Y$ is finite, then we show that

$$
\sup \mathbf{L} \Lambda^{\mathfrak{a}}\left(X \otimes_{R}^{\mathbf{L}} Y\right) \leq \operatorname{Gfd}_{R} X+\sup Y .
$$

Finally, we prove that a Gorenstein local ring $(R, \mathfrak{m})$ is regular if and only if the $\mathfrak{m}$-adic completion of any Gorenstein flat $R$-module is flat.

\section{THE RESULTS}

For proving our main result, we need the following three lemmas. For an ideal $\mathfrak{a}$ of $R, \mathrm{~cd}_{\mathfrak{a}}(R)$ denotes the supremum of $i$ 's such that $i$-th local cohomology module of $R$ with respect to $\mathfrak{a}$ is nonzero.

Lemma 2.1. Let $\mathfrak{a}$ be an ideal of the Noetherian ring $R$ and $X \in \mathcal{D}_{\square}(R)$. Then

$$
\sup \mathbf{L} \Lambda^{\mathfrak{a}}(X) \leq \sup X+\operatorname{cd}_{\mathfrak{a}}(R) .
$$

Proof. Let $\underline{\mathfrak{a}}$ be a set of generators of $\mathfrak{a}$. Then, we have $\mathbf{L} \Lambda^{\mathfrak{a}}(X) \simeq \mathbf{R} \operatorname{Hom}_{R}(\check{C}(\underline{\mathfrak{a}}), X)$. Hence, $\mathrm{C}$, Proposition A.4.6] implies that

$$
\sup \mathbf{L} \Lambda^{\mathfrak{a}}(X) \leq \sup X-\inf \check{C}(\underline{\mathfrak{a}})=\sup X+\operatorname{cd}_{\mathfrak{a}}(R) .
$$

Let $\mathfrak{a}$ be an ideal of $R$. Simon [Si, Section 5.1] investigated the class $C_{\mathfrak{a}}$ of $R$-modules $M$ for which $H_{i}^{\mathfrak{a}}(M)=0$ for all $i>0$, and such that the natural homomorphism $H_{0}^{\mathfrak{a}}(M) \longrightarrow \Lambda^{\mathfrak{a}}(M)$ is an isomorphism. By [M, Corllary 4.5] every flat $R$-module belongs to $C_{\mathfrak{a}}$. Next, we improves this result of Matlis by showing that every Gorenstein flat $R$-module belongs to $C_{\mathfrak{a}}$.

Lemma 2.2. Let $\mathfrak{a}$ be an ideal of the Noetherian ring $R$ and $Q$ a Gorenstein flat $R$-module. 
i) $Q$ is $\Lambda^{\mathfrak{a}}$-acyclic.

ii) There is a natural $R$-isomorphism $H_{0}^{\mathfrak{a}}(Q) \cong \Lambda^{\mathfrak{a}}(Q)$.

Proof. i) There exists an exact sequence

$$
F=\cdots \rightarrow F_{1} \rightarrow F_{0} \rightarrow F_{-1} \rightarrow F_{-2} \rightarrow \cdots
$$

of flat $R$-modules such that $Q \cong \operatorname{im}\left(F_{0} \rightarrow F_{-1}\right)$. Set $L_{0}:=Q$ and $L_{i}:=\operatorname{im}\left(F_{i} \rightarrow F_{i-1}\right)$ for all $i<0$. For each $i<0$, the exact sequence

$$
0 \rightarrow L_{i+1} \rightarrow F_{i} \rightarrow L_{i} \rightarrow 0,
$$

yields the following long exact sequence of local homology modules

$$
\cdots \rightarrow H_{j+1}^{\mathfrak{a}}\left(F_{i}\right) \rightarrow H_{j+1}^{\mathfrak{a}}\left(L_{i}\right) \rightarrow H_{j}^{\mathfrak{a}}\left(L_{i+1}\right) \rightarrow H_{j}^{\mathfrak{a}}\left(F_{i}\right) \rightarrow \cdots .
$$

The argument of $\left[\mathrm{M}\right.$, Corollary 4.5] yields that any flat $R$-module is $\Lambda^{\mathfrak{a}}$-acyclic. Hence, we conclude the isomorphisms $H_{j}^{\mathfrak{a}}\left(L_{i+1}\right) \cong H_{j+1}^{\mathfrak{a}}\left(L_{i}\right)$ for all $i<0$ and all $j \geq 1$. Let $n:=\operatorname{cd}_{\mathfrak{a}}(R)$. Then by Lemma 2.1, one has

$$
H_{j}^{\mathfrak{a}}(Q) \cong H_{j+1}^{\mathfrak{a}}\left(L_{-1}\right) \cong \ldots \cong H_{j+n}^{\mathfrak{a}}\left(L_{-n}\right)=0
$$

for all $j>0$.

ii) By [Si, Section 5.1], there is a natural transformation of functors $\xi: H_{0}^{\mathfrak{a}}(\cdot) \rightarrow \Lambda^{\mathfrak{a}}(\cdot)$, which is such that $\xi_{N}$ is surjective for all $R$-modules $N$. The proof of [M, Corollary 4.5] implies that $\xi_{F}$ is an isomorphism for any flat $R$-module $F$. From the definition of Gorenstein flat $R$-modules, one can construct an exact sequence $0 \rightarrow Q \stackrel{f}{\rightarrow} F \stackrel{g}{\rightarrow} N \rightarrow 0$ of $R$-modules and $R$-homomorphisms in which $F$ is flat and $N$ is Gorenstein flat. By i), we deduce the following exact sequence

$$
0 \rightarrow H_{0}^{\mathfrak{a}}(Q) \stackrel{H_{0}^{\mathfrak{a}}(f)}{\longrightarrow} H_{0}^{\mathfrak{a}}(F) \stackrel{H_{0}^{\mathfrak{a}}(g)}{\longrightarrow} H_{0}^{\mathfrak{a}}(N) \rightarrow 0 .
$$

Now, from the commutative square

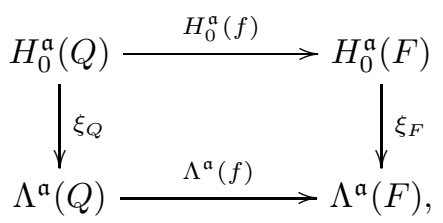

it becomes clear that $\xi_{Q}$ is an isomorphism, as required.

Next, we record the following immediate corollary of Lemma 2.2.

Corollary 2.3. Let $\mathfrak{a}$ be an ideal of the Noetherian ring $R$. The functor $\Lambda^{\mathfrak{a}}(-)$ is exact on the full subcategory of Gorenstein flat R-modules.

The following useful lemma is well-known, and so we skip its proof.

Lemma 2.4. Let $T: \mathcal{C}_{0}(R) \rightarrow \mathcal{C}_{0}(R)$ be a covariant additive functor. Any morphism of complexes $\alpha: X \rightarrow Y$ yields an isomorphism of complexes $\phi_{\alpha}$ : Cone $(T(\alpha)) \longrightarrow T(\operatorname{Cone}(\alpha))$.

Next, we present our main result. 
Theorem 2.5. Let $\mathfrak{a}$ be an ideal of the Noetherian ring $R$. Let $X \in \mathcal{D}_{\sqsupset}(R)$ and $Q$ a bounded to the right complex of Gorenstein flat $R$-modules such that $Q \simeq X$. Then $\mathbf{L} \Lambda^{\mathfrak{a}}(X) \simeq \Lambda^{\mathfrak{a}}(Q)$, and so $H_{i}^{\mathfrak{a}}(X)=H_{i}\left(\Lambda^{\mathfrak{a}}(Q)\right)$ for all $i \in \mathbb{Z}$. In particular, $\sup \mathbf{L} \Lambda^{\mathfrak{a}}(X) \leq \operatorname{Gfd}_{R} X$.

Proof. Let $P$ be a projective resolution of $X$. Then $P \simeq Q$, and hence [AF, 1.1.P and 1.4.P] and [C, A.4.1] yield the existence of a quasi-isomorphism $\alpha: P \rightarrow Q$. Now, Cone $(\alpha)$ is an exact bounded to the right complex of Gorenstein flat $R$-modules. By splitting Cone $(\alpha)$ into short exact sequences and using $[\mathrm{H}$, Proposition 3.12] and Corollary 2.3, we see that $\Lambda^{\mathfrak{a}}(\operatorname{Cone}(\alpha))$ is exact, and so by Lemma 2.4, $\operatorname{Cone}\left(\Lambda^{\mathfrak{a}} \alpha\right)$ is also exact. Therefore $\Lambda^{\mathfrak{a}}(\alpha): \Lambda^{\mathfrak{a}}(P) \rightarrow \Lambda^{\mathfrak{a}}(Q)$ is a quasi-isomorphism, and so

$$
\mathbf{L} \Lambda^{\mathfrak{a}}(X) \simeq \Lambda^{\mathfrak{a}}(P) \simeq \Lambda^{\mathfrak{a}}(Q) .
$$

Corollaries 2.8 and 2.10 are the main applications of this theorem. To prove Corollary 2.8, we need a couple of lemmas. The first lemma slightly improves [FI, 1.10]. Recall that for a complex $X \in \mathcal{D}_{\sqsupset}(R)$, any bounded to the right complex $F$ such that $F$ consists of flat $R$-modules and there exists a quasi-isomorphism $\alpha: F \longrightarrow X$ is called a flat resolution of $X$.

Lemma 2.6. Let $\mathfrak{a}$ be an ideal of the Noetherian ring $R$ and $X, Y \in \mathcal{D}_{\sqsupset}(R)$. Let $Q$ be a bounded to the right complex of Gorenstein flat $R$-modules such that $Q \simeq X$ and $F$ a flat resolution of $Y$. Then $\mathbf{L} \Lambda^{\mathfrak{a}}\left(X \otimes_{R}^{\mathbf{L}} Y\right) \simeq \Lambda^{\mathfrak{a}}\left(Q \otimes_{R} F\right)$. Moreover, if $X$ is homologically bounded and all homology modules of $Y$ are finitely generated, then $\mathbf{L} \Lambda^{\mathfrak{a}}\left(X \otimes_{R}^{\mathbf{L}} Y\right) \simeq \mathbf{L} \Lambda^{\mathfrak{a}}(X) \otimes_{R}^{\mathbf{L}} Y$.

Proof. By [CH, Ascent table II a)] $Q \otimes_{R} F$ is a complex of Gorenstein flat $R$-modules. Hence, as the complex $Q \otimes_{R} F$ is bounded to the right and $Q \otimes_{R} F \simeq X \otimes_{R}^{\mathbf{L}} Y$, Theorem 2.5 implies that $\mathbf{L} \Lambda^{\mathfrak{a}}\left(X \otimes_{R}^{\mathbf{L}} Y\right) \simeq \Lambda^{\mathfrak{a}}\left(Q \otimes_{R} F\right)$.

Now, assume that all homology modules of $Y$ are finitely generated. Since, by [CFH, 5.8], $\check{C}(\underline{\mathfrak{a}})$ has finite projective dimension, $[\mathrm{CH}$, Proposition 2.2 vi)] yields that

$$
\mathbf{L} \Lambda^{\mathfrak{a}}\left(X \otimes_{R}^{\mathbf{L}} Y\right) \simeq \mathbf{R} \operatorname{Hom}_{R}\left(\check{C}(\underline{\mathfrak{a}}), X \otimes_{R}^{\mathbf{L}} Y\right) \simeq \mathbf{R} \operatorname{Hom}_{R}(\check{C}(\underline{\mathfrak{a}}), X) \otimes_{R}^{\mathbf{L}} Y \simeq \mathbf{L} \Lambda^{\mathfrak{a}}(X) \otimes_{R}^{\mathbf{L}} Y .
$$

Recall that the large restricted flat dimension of an $R$-module $M$ is defined by

$$
\operatorname{Rfd}_{R} M:=\sup \left\{i \in \mathbb{N}_{0} \mid \operatorname{Tor}_{i}^{R}(M, T) \neq 0 \text { for some R-module } T \text { with finite flat dimension }\right\} .
$$

Lemma 2.7. Let $\mathfrak{a}$ be an ideal of the Noetherian ring $R$ and $Q$ a Gorenstein flat $R$-module. Then $\operatorname{Rfd}_{R} \Lambda^{\mathfrak{a}}(Q)=0$. Moreover, if $R$ possesses a dualizing complex, then $\Lambda^{\mathfrak{a}}(Q)$ is Gorenstein flat.

Proof. Since $Q$ is Gorenstein flat, there exists an exact sequence

$$
X=0 \rightarrow Q \rightarrow F_{-1} \rightarrow F_{-2} \rightarrow \cdots \rightarrow F_{-s} \rightarrow \cdots,
$$

where each $F_{i}$ is flat and $K_{i}:=\operatorname{im}\left(F_{-i} \rightarrow F_{-(i+1)}\right)$ is Gorenstein flat for all $i \geq 1$. Let $T$ be an $R$-module of finite flat dimension $s$ 'say. By Corollary 2.3, the functor $\Lambda^{\mathfrak{a}}(-)$ is exact on the full subcategory of Gorenstein flat $R$-modules. Hence, we have the following short exact sequences

$$
0 \rightarrow \Lambda^{\mathfrak{a}}(Q) \rightarrow \Lambda^{\mathfrak{a}}\left(F_{-1}\right) \rightarrow \Lambda^{\mathfrak{a}}\left(K_{1}\right) \rightarrow 0
$$


and

$$
0 \rightarrow \Lambda^{\mathfrak{a}}\left(K_{i}\right) \rightarrow \Lambda^{\mathfrak{a}}\left(F_{-(i+1)}\right) \rightarrow \Lambda^{\mathfrak{a}}\left(K_{i+1}\right) \rightarrow 0
$$

for all $i \geq 1$. By $\left[\mathrm{B}\right.$, 1.4.7], $\Lambda^{\mathfrak{a}}(F)$ is flat for all flat $R$-modules $F$. Therefore, using the above short exact sequences successively yields that

$$
\operatorname{Tor}_{j}^{R}\left(\Lambda^{\mathfrak{a}}(Q), T\right) \cong \operatorname{Tor}_{j+1}^{R}\left(\Lambda^{\mathfrak{a}}\left(K_{1}\right), T\right) \cong \ldots \cong \operatorname{Tor}_{j+s}^{R}\left(\Lambda^{\mathfrak{a}}\left(K_{s}\right), T\right)=0,
$$

for all $j \geq 1$. This shows that $\operatorname{Rfd}_{R} \Lambda^{\mathfrak{a}}(Q)=0$.

Now, assume that $R$ possesses a dualizing complex. By Theorem $2.5, \mathbf{L} \Lambda^{\mathfrak{a}}(Q) \simeq \Lambda^{\mathfrak{a}}(Q)$, and so by CFH, Theorem $5.10 \mathrm{~b})], \operatorname{Gfd}_{R} \Lambda^{\mathfrak{a}}(Q)$ is finite. Thus, by [H, Theorem 3.19], it turns out that $\operatorname{Gfd}_{R} \Lambda^{\mathfrak{a}}(Q)=$ $\operatorname{Rfd}_{R} \Lambda^{\mathfrak{a}}(Q)=0$, and so $\Lambda^{\mathfrak{a}}(Q)$ is Gorenstein flat.

Part i) of the following corollary strengthens [CFH, Theorem $5.10 \mathrm{~b}$ )].

Corollary 2.8. Let $R$ be a Noetherian ring possessing a dualizing complex and $\mathfrak{a}$ an ideal of $R$.

i) Let $X \in \mathcal{D}_{\sqsupset}(R)$. Then $\operatorname{Gfd}_{R} \mathbf{L} \Lambda^{\mathfrak{a}}(X) \leq \operatorname{Gfd}_{R} X$.

ii) Let $Y \in \mathcal{D}_{\square}^{f}(R)$ be a complex such that either its projective or injective dimension is finite and $X \in \mathcal{D}_{\square}(R)$. Then $\sup \mathbf{L} \Lambda^{\mathfrak{a}}\left(X \otimes_{R}^{\mathbf{L}} Y\right) \leq \operatorname{Gfd}_{R} X+\sup Y$.

Proof. i) It follows by Theorem 2.5 and Lemma 2.7 .

ii) The inequality certainly holds if either $X$ has infinite Gorenstein flat dimension or $Y$ is exact. So, assume that the Gorenstein flat dimension of $X$ is finite and $Y$ is non-exact. By i), the Gorenstein flat dimension of $\mathbf{L} \Lambda^{\mathfrak{a}}(X)$ is finite, hence Lemma 2.6 and $[\mathrm{CFH}$, Theorem 3.5] yield that

$$
\begin{aligned}
\sup \mathbf{L} \Lambda^{\mathfrak{a}}\left(X \otimes_{R}^{\mathfrak{L}} Y\right) & =\sup \left(\mathbf{L} \Lambda^{\mathfrak{a}}(X) \otimes_{R}^{\mathfrak{L}} Y\right) \\
& \leq \operatorname{Gfd}_{R} \mathbf{L} \Lambda^{\mathfrak{a}}(X)+\sup Y \\
& \leq \operatorname{Gfd}_{R} X+\sup Y .
\end{aligned}
$$

Lemma 2.9. Let $R$ be a Noetherian ring possessing a dualizing complex and $\mathfrak{a}$ an ideal of $R$. The following are equivalent:

i) $\Lambda^{\mathfrak{a}}(Q)$ is flat for all Gorenstein flat $R$-module $Q$.

ii) $\operatorname{Gfd}_{R} Q=\mathrm{fd}_{R} Q$ for all $\mathfrak{a}$-adic complete $R$-modules $Q$.

Proof. $i) \Rightarrow i i)$ Let $N$ be a a-adic complete $R$-module. We have to show that $\operatorname{Gfd}_{R} N=\operatorname{fd}_{R} N$. Since, by [H, Theorem 3.19], $\operatorname{Gfd}_{R} N \leq \operatorname{fd}_{R} N$, we can assume that $n:=\operatorname{Gfd}_{R} N$ is finite. By [Si, Proposition 2.5] and its proof, one can choose a flat resolution

$$
F=\cdots \rightarrow F_{i} \stackrel{d_{i}}{\rightarrow} F_{i-1} \rightarrow \cdots \rightarrow F_{0} \rightarrow 0
$$

of $N$ such that $F_{i}$ and $\operatorname{ker} d_{i+1}$ are a-adic complete for all $i \geq 0$. Let $Q:=\operatorname{ker} d_{n-1}$. Then $Q$ is $\mathfrak{a}$-adic complete and by [ $\mathrm{H}$, Theorem 3.14], $Q$ is Gorenstein flat. Thus i) implies that $Q$ is flat, and so $N$ has finite flat dimension. Now, use [ $\underline{\mathrm{H}}$, Theorem 3.19] again to deduce that $\operatorname{Gfd}_{R} N=\mathrm{fd}_{R} N$.

$i i) \Rightarrow i$ ) Let $Q$ be a Gorenstein flat $R$-module. Then, by Lemma 2.7 , the $\mathfrak{a}$-adic complete $R$-module $\Lambda^{\mathfrak{a}}(Q)$ is Gorenstein flat. Hence, by ii), it turns out that $\operatorname{fd}_{R} \Lambda^{\mathfrak{a}}(Q)=\operatorname{Gfd}_{R} \Lambda^{\mathfrak{a}}(Q)=0$, and so $\Lambda^{\mathfrak{a}}(Q)$ is flat.

Next, we present a characterization of regularity of Gorenstein local rings. 
Corollary 2.10. Let $(R, \mathfrak{m}, k)$ be a local Gorenstein ring. The following are equivalent:

i) $\Lambda^{\mathfrak{m}}(Q)$ is flat for all Gorenstein flat $R$-modules $Q$.

ii) $\operatorname{Gfd}_{R} Q=\mathrm{fd}_{R} Q$ for all $\mathfrak{m}$-adic complete $R$-modules $Q$.

iii) $R$ is regular.

Proof. i) and ii) are equivalent by Lemma 2.9 .

Since $R$ is Gorenstein, we see that $R$ is a dualizing complex of $R$ and $k$ has finite Gorenstein flat dimension. Recall that $R$ is regular if and only if the flat dimension of the $\mathfrak{m}$-adic complete $R$-module $k$ is finite and if and only if the flat dimension of any $R$-module is finite. Thus [ $\underline{H}$, Theorem 3.19] implies that ii) and iii) are equivalent.

We thank anonymous referee for suggesting the following example.

Example 2.11. In the above corollary, the assumption of Gorensteiness of $R$ can not be deleted. To this end, let $(R, \mathfrak{m})$ be a non-Gorenstein local ring such that $\mathfrak{m}^{2}=0$, for examples of such rings see [CH] Example 4.2]. Then by [CH, Proposition 4.3] any Gorenstein flat $R$-module is free, and so by [B, 1.4.7], $\Lambda^{\mathfrak{m}}(Q)$ is flat for all Gorenstein flat $R$-modules $Q$.

\section{REFERENCES}

[AF] L. Avramov and H-B. Foxby, Homological dimensions of unbounded complexes, J. Pure Appl. Algebra, 71(2-3), (1991), 129-155.

[B] J. Bartijn, Flatness, completions, regular sequences un ménage à trois, Thesis, Utrecht, 1985.

[C] L.W. Christensen, Gorenstein dimensions, Lecture Notes in Mathematics, 1747, Springer-Verlag, Berlin, 2000.

$[\mathrm{CFH}]$ L.W. Christensen, A. Frankild and H. Holm, On Gorenstein projective, injective and flat dimensions-a functorial description with applications, J. Algebra, 302(1), (2006), 231-279.

[CH] L.W. Christensen and H. Holm, Ascent properties of Auslander categories, Canad. J. Math., 61(1), (2009), 76-108.

[EJT] E.E. Enochs, O.M.G Jenda and B. Torrecillas, Gorenstein flat modules, Nanjing Daxue Xuebao Shuxue Bannian Kan 10(1), (1993), 1-9.

[FI] H-B. Foxby and S. Iyengar, Depth and amplitude for unbounded compelexes, Commutative algebra (Grenoble/Lyon, 2001), 119-137, Contemp. Math., 331, Amer. Math. Soc., Providence, RI, 2003.

[Fr] A. Frankild, Vanishing of local homology, Math. Z., 244(3), (2003), 615-630.

[GM] J.P.C. Greenlees and J.P. May, Derived functors of I-adic completion and local homology, J. Algebra, 149(2), (1992), 438-453.

[H] H. Holm, Gorenstein homological dimensions, J. Pure Appl. Algebra, 189(1-3), (2004), 167-193.

[LLT] J. Lipman, A.J. López and L.A. Tarrio, Local homology and cohomology on schemes, Ann. Sci. École Norm. Sup., (4)30(1), (1997), 1-39.

[M] E. Matlis, The Koszul complex and duality, Comm. Algebra, 1, (1974), 87-144.

[Sa] R. Sazeedeh, Gorenstein injective modules and local cohomology, Proc. Amer. Math. Soc., 132(10), (2004), $2885-2891$.

[Sc] P. Schenzel, Proregular sequences, local cohomology, and completion, Math. Scand., 92(2), (2003), 161-180.

[Si] A-M. Simon, Some homological properties of complete modules, Math. Proc. Cambridge Philos. Soc., 108(2), (1990), 231-246.

F. Mohammadi Aghjeh Mashhad, Science and Research Branch, Islamic Azad University, Tehran, Iran.

E-mail address: mohammadi_fh@yahoo.com

K. Divaani-Aazar, Department of Mathematics, Az-Zahra University, Vanak, Post Code 19834, Tehran, Iran.

E-mail address: kdivaani@ipm.ir 